\title{
Technical challenges during therapeutic ERCP in a patient with Situs Inversus Totalis
}

\author{
Shruti Khurana, Hani Zamil, Erik Rahimi, Sushovan Guha and Nirav Thosani* \\ Division of Gastroenterology, Hepatology and Nutrition, Ertan Digestive Disease Center, The University of Texas \\ Health Science Center at Houston, Houston, Texas, USA
}

Received: July 12, 2016; Accepted: October 18, 2016; Published: May 4, 2017

*Corresponding author: Nirav Thosani, Division of Gastroenterology, Hepatology and Nutrition, Ertan Digestive Disease Center, The University of Texas Health Science Center at Houston, Houston, Texas, USA, Tel: 713-500-6677; Fax: 713-500-6699; E-mail: Nirav.Thosani@uth.tmc.edu

\section{Abstract \\ Situs inversus totalis is an extremely rare autosomal recessive disorder occurring in $0.01 \%$ of the population. The reversal of visceral organs poses technical difficulties for therapeutic intervention during endoscopic retrograde cholangiopancreatography (ERCP). We describe a unique case of a patient with situs inversus who underwent therapeutic ERCP for management of choledocholithiasis.}

\section{Case Study}

A 37-year-old woman with a history of situs inversus totalis, complex congenital cyanotic heart disease, polysplenia and laparoscopic partial cholecystectomy performed 10 years prior, presented with post-prandial right upper quadrant pain and nausea. She had elevated bilirubin, and computed tomography (CT) scan showed cholelithiasis in the remnant gallbladder. An endoscopic retrograde cholangiopancreatography (ERCP) was performed for possible choledocholithiasis. Patient was placed in the left oblique position. There was altered anatomy of the gastric curvature and the ampulla was located in the 2 o'clock position. Biliary access was achieved by keeping the scope in the long position, rotating the duodenoscope 1800 , and rotating the sphincterotome to the right side. Cholangiogram showed a mirror image of the transposed biliary tree and remnant gallbladder with filling defectsconsistent with cholelithiasis. Biliary sphincterotomy was performed and balloon sweep showed biliary stone fragments, consistent with choledocholithiasis. She subsequently underwent cholecystectomy for removal of the remnant gallbladder.

Situs inversus is a rare benign congenital anomaly that may pose difficulty during ERCP [1]. Different techniques have been used for biliary access in patients with situs inversus such as, a "mirror image" ERCP technique with the endoscopic maneuvers performed in an inverse fashion, or positioning the patient lying prone on her right side [2,3]. In our case biliary access is easily achievable in patients with situs inversus by keeping the patient in the left oblique position, along with maneuvering the duodenoscope and sphincterotome towards the inversed position of the ampulla.

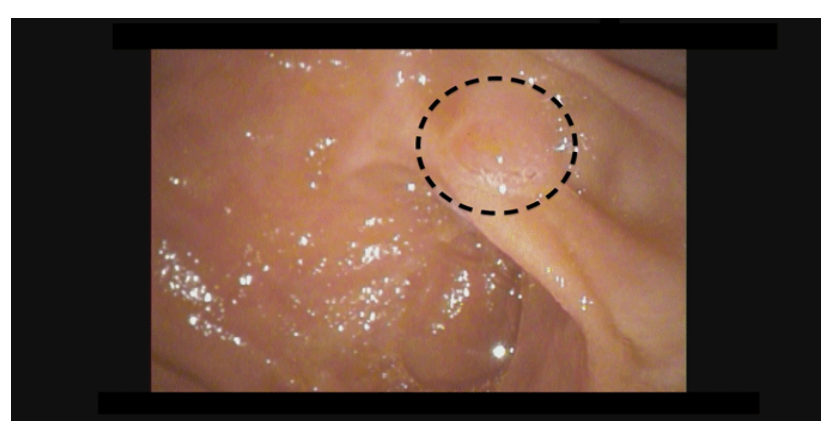

Figure 1: Endoscopic view of the major papilla

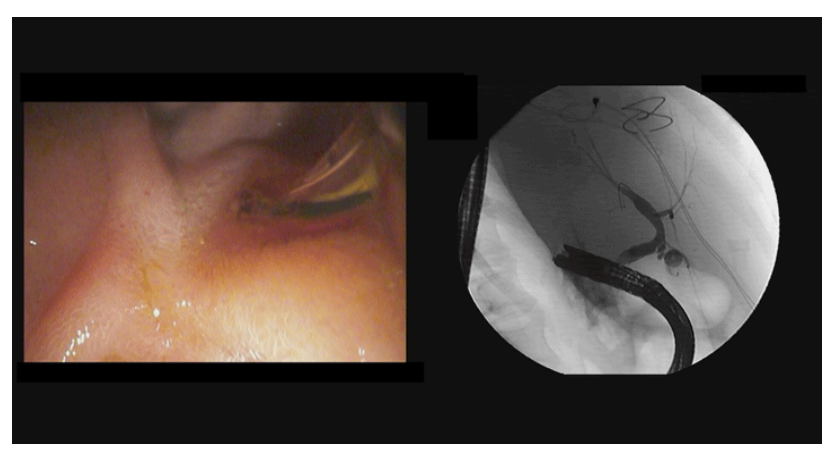

Figure 2: ERCP showing (A) Endoscopic view of bile duct cannulation; and (B) fluoroscopic image of the biliary tree and remnant gallbladder in an inverse position

\section{References}

1. Morelli SH, Young L, Reid B, Ruttenberg H, Bamshad MJ. Clinical analysis of families with heart, midline, and laterality defects. Am J Med Genet. 2001;101(4):388-392.

2. Nordback I, Airo I. ERCP and endoscopic papillotomy in complete abdominal situs inversus. Gastrointest Endosc. 1988;34(2):150.

3. García-Fernández FJ, Infantes JM, Torres Y, Mendoza FJ, Alcazar FJ. ERCP in complete situs inversus viscerum using a "mirror image" technique. Endoscopy. 2010;42 (Suppl. 02): E316 - E317. doi: 10.1055/s-0030-1255813 